\title{
QUANTAL NATURE OF TRANSMISSION AT THE SYNAPSE BETWEEN HAIR CELLS AND EIGHTH NERVE FIBERS
}

\author{
Y. Ishit, S. MatsuUra and T. Furukawa \\ Department of Physiology, Osaka City University Medical School \\ Abeno-ku, Osaka 545, Japan
}

\begin{abstract}
Summary 1. An intracellular recording technique was used to record excitatory postsynaptic potentials (EPSP's) from the eighth nerve fibers of goldfish. EPSP's were evoked by applying sound stimulus, and spontaneous miniature EPSP's were also observed.

2. Even when the stimulus sound was kept at a fixed intensity level, the EPSP amplitude showed some fluctuations including occasional failures of the response.

3. The applicability of Poisson's law was tested for several intensity levels of sound stimulus in the same fibers. The Poisson relation was found applicable to the amplitude distribution of EPSP's evoked by a weak sound, but some discrepancies were found for a strong sound.

4. It was concluded that the transmission between hair cells and primary eighth nerve fibers of goldfish occurred in quantal steps. Some theoretical implications of the present results were discussed.
\end{abstract}

It has been shown in previous reports (FurUKAWA and IsHII, 1967) that the excitatory transmission from hair cells to certain eighth nerve fibers in the goldfish sacculus takes place with mechanisms largely similar to those in other well known chemical synapses (KATZ, 1966). For example, the presence of a definite synaptic delay and the occurrence of spontaneous miniature EPSP's have been demonstrated. Further, EPSP's evoked by a repetitive application of the same sound stimulus often show some fluctuations in their size. Especially when the EPSP's are small in size, the fluctuation may become so remarkable that it reminiscent of the quantal fluctuation of postsynaptic potentials as observed in other structures (MARTIN, 1965).

In the present paper, we examined the possibility of applying the Poisson analysis to the present case. The condition under which we observed the EPSP fluctuation, however, was a little different from that commonly used. EPSP's

Received for publication January 6, 1971

石井義久, 松裏修四, 古河太郎 
were reduced in size in the present case by applying a very weak sound, while the more usual procedure is to reduce the quantal content of the transmitter release by reducing the $\mathrm{Ca}^{2+}$ content of the medium or by elevating its $\mathrm{Mg}^{2+}$ content.

\section{METHODS}

Experiments were carried out on live and immobilized goldfish about $12 \mathrm{~cm}$ in size. Nerve fibers from the sacculus, i.e., the inner ear of the goldfish, consist of two groups of fibers of different diameters. All the experiments in the present study were carried out on the large fiber group, i.e., our S1 fibers (d: $15 \mu)$. Potentials were recorded intracellularly with a glass micropipette electrode and a reference electrode of chlorinated silver wire placed in contact with brain tissue. Micropipettes were filled with $3 \mathrm{M} \mathrm{KCl}$ and their tip diameter was $1 \mu$ or less. As a sound stimulus, tone pips were delivered through a loudspeaker placed about $30 \mathrm{~cm}$ from the fish. Sound intensity was varied with two attenuators, one of which had $5 \mathrm{~dB}$ steps and the other $1 \mathrm{~dB}$ steps. The absolute level of sound intensity was checked with a condenser microphone and expressed in reference to the sound pressure level (SPL). Tone pips had a duration of 10-20 msec and were usually applied at a rate of two times per sec. Experiments were carried out at room temperature. Experimental methods including operation and other recording procedures have been reported elsewhere (FURUKAWA and IsHII, 1967).

\section{RESULTS}

\section{Fluctuations of the EPSP size}

Figure 1 shows an example in which EPSP's to the same sound stimulus showed a very marked fluctuation. The fluctuation was especially marked in the EPSP's that were evoked in response to the first compression phase of the stimulus sound. This fiber is obviously responding to both compression and rarefaction phases of the sound, but responses to the former are much greater than those to the latter. The response to the second compression phase of the sound developed into a spike and was much greater than other responses. As will be shown in Figs. 2 and 3, fluctuation in the EPSP size were generally more marked for a weak stimulus than for a strong one. In the case of Fig. 1 too, fluctuation in size of the first EPSP's is much more marked perhaps because the stimulus intensity for them is weaker than for latter ones. Figure 2 shows two cases in which fluctuation of EPSP size was examined for two different sound intensities. The fiber shown in A is responding only to the compression phases of the sound (see A3). As shown in A1, a random discharge of spontaneous miniature EPSP's was observed in this fiber. Responses to a weak stimulus sound, shown in A2, were very irregular, but the randomness was much less marked in A3 where the sound 


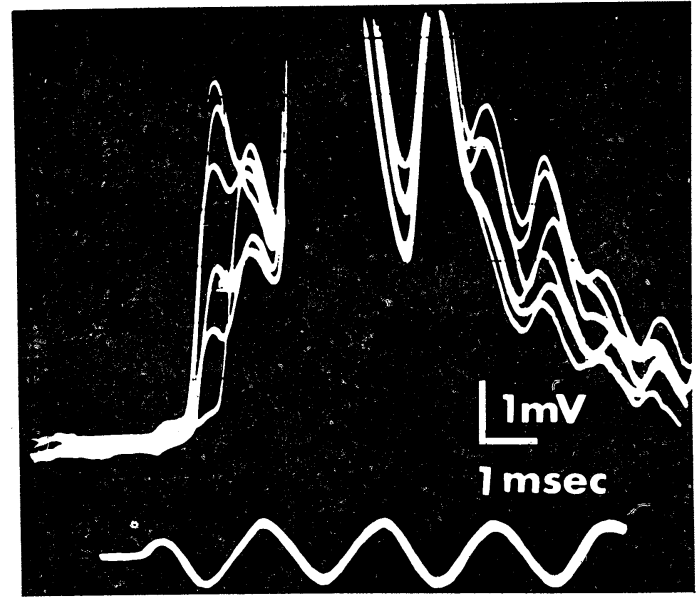

Fig. 1. Sound-evoked EPSP's recorded intracellularly from an eighth nerve fiber. Note that the fluctuation in size is much more marked in the first EPSP's than the rest of them. Upward deflections in the sound monitor (lower trace) indicate compression phases of the sound. Stimulus: $300 \mathrm{~Hz}$ and $85 \mathrm{~dB}$.
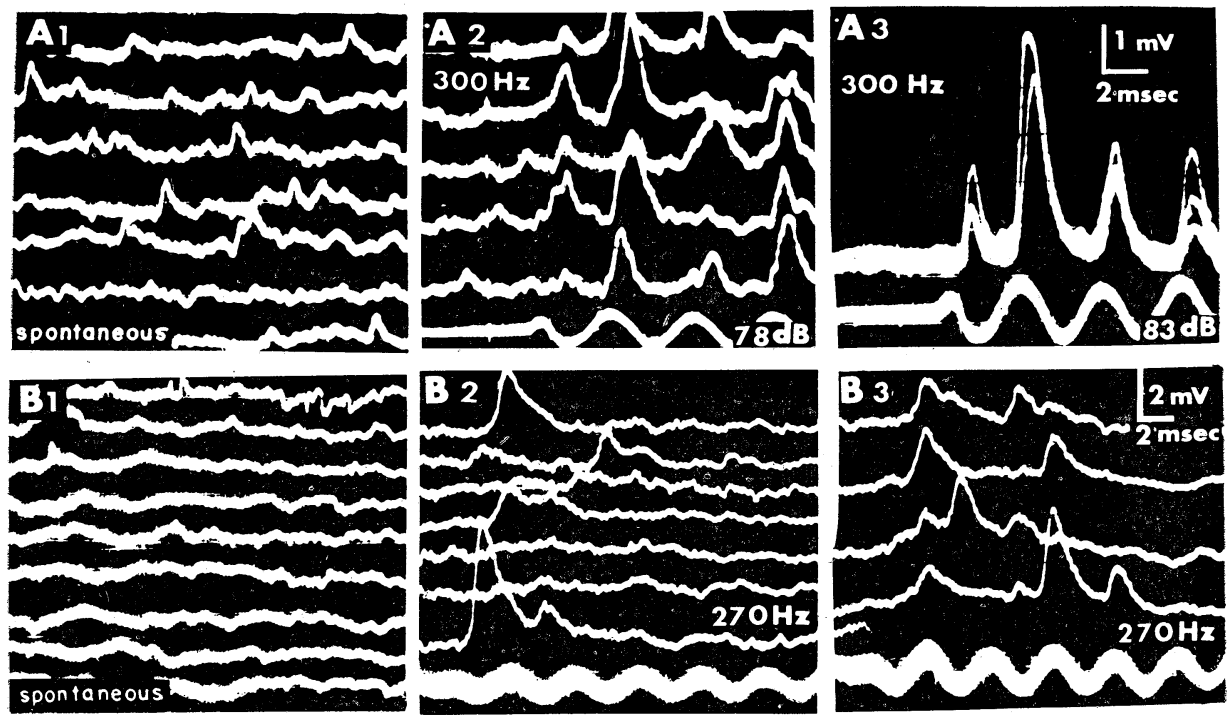

Fig. 2. Spontaneous and evoked EPSP's in two fibers. Discharge of spontaneous miniature EPSP's was very frequent in the fiber $\mathrm{A}$ (see $\mathrm{Al}$ ), but was almost nonexistent in the fiber B. Stimulus sound: $300 \mathrm{~Hz}, 78$ and $83 \mathrm{~dB}$ in $\mathrm{A} 2$ and $\mathrm{A} 3 ; 270 \mathrm{~Hz}, 78$ and $83 \mathrm{~dB}$ in $\mathrm{B} 2$ and $\mathrm{B} 3$, respectively. 
intensity was increased by $5 \mathrm{~dB}$. Despite their much larger size, EPSP's in A3 have a short duration, their half width being less than $1 \mathrm{msec}$, indicating that the release of the transmitter packet quanta took place with better synchronization in A3 than in A2 (FURUKAwA et al., in preparation). Figure 2B shows an instance in which the discharge of miniature EPSP's was so infrequent that they could not be recorded at all (see B1). This fiber was also responding to the compression as well as to the rarefaction phases of the sound. B2 and B3 show that there were very marked fluctuations in the EPSP size in this fiber.

\section{Statistical analysis of the fluctuation in the EPSP size}

When the release of the transmitter at a neuromuscular junction is suppressed by adding $\mathrm{Mg}^{2+}$ to the medium and/or by removing $\mathrm{Ca}^{2+}$ from it, the amplitude of the end-plate potential shows a marked fluctuation. The distribution of EPSP size at such a junction has been shown to obey Poisson's law, indicating that there are large numbers of contributing units at the junction, each with a small probability of response to nerve impulses (KATZ, 1966; MARTIN, 1965). Since, as is shown in Figs. 1 and 2, there were marked fluctuations in the size of EPSP's in the present material, some efforts were made to see whether a similar type of statistical analysis could be applied to describe the phenomena. However, our analysis has been very limited in extent due to the very infrequent occurrence of spontaneous miniature EPSP's and also due to technical difficulties in obtaining stable intracellular records. In the present investigation, we tried to calculate the distributions of EPSP size which would be expected from the statistical theory and tried to compare them with those observed (KATZ, 1966; MARTIN, 1965).

There are several different methods for calculating the mean quantum content $m$, i.e., the average number of the quanta released per stimulus during a series of trials. In cases where miniature EPSP's could be recorded, $m$ may be obtained by dividing the average amplitude of the EPSP's in the series by the average amplitude of spontaneous EPSP's, namely

$$
m=\frac{\text { mean amplitude of EPSP responses }(v)}{\text { mean amplitude of spontaneous potentials }\left(v_{1}\right)}
$$

Using $m$ thus obtained, the expected number of failures, single unit responses and multiple responses could be calculated respectively based on the Poisson equation.

Figure 3 shows responses of a fiber on which the statistical analysis was carried out. Miniature EPSP's shown in A have a typical time course with a duration of $0.6-0.8 \mathrm{msec}$ as measured at half their height (see also Fig. 2 A1). The histogram shown in Fig. 4D illustrates the size distribution of these spontaneous potentials. The mean amplitude of these spontaneous potentials, i.e., the mean quantum size $v_{1}$, was $0.43 \mathrm{mV}$ and its standard deviation 

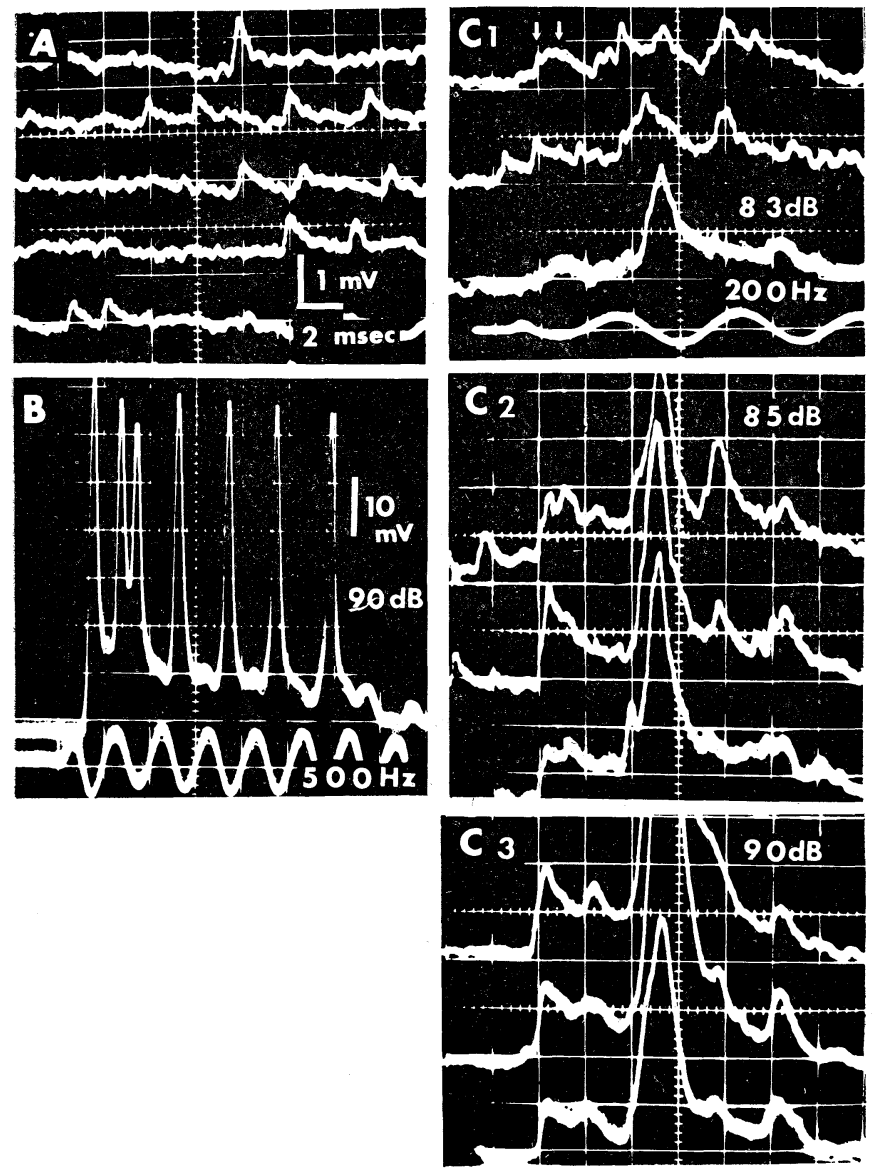

Fig. 3. Sample responses of a S1 fiber. A: Spontaneous miniature EPSP's. B: Spike train evoked by a strong sound at $500 \mathrm{~Hz}$ and $90 \mathrm{~dB}$. C: Responses to weak sounds at $200 \mathrm{~Hz}$ and 83,85 , and $90 \mathrm{~dB}$ for $\mathrm{C} 1$ to $\mathrm{C} 3$, respectively.

was $0.11 \mathrm{mV}$. Figure 3B shows the response of this fiber to a strong sound at $500 \mathrm{~Hz}$. Although EPSP's and spike potentials were set up in response to each compression phase of the sound, small EPSP's were evoked also in response to rarefaction phases. Therefore, this fiber responds more strongly to compression phases but also responds weakly to rarefaction phases. This is also observed in records of $\mathrm{C} 1-\mathrm{C} 3$ in which weak sounds at $200 \mathrm{~Hz}$ were applied. The sound intensity was $83 \mathrm{~dB}$ SPL in $\mathrm{C} 1$ and was increased by 2 and $7 \mathrm{~dB}$ in $\mathrm{C} 2$ and $\mathrm{C} 3$, respectively. The statistical analysis was performed on the first responses that corresponded to the initial transient-like deflection in the sound monitor. As can be seen in $\mathrm{Cl}$, there was a considerable fluctuation not only in the response size but also in the time span of the response. The latter factor made it difficult 

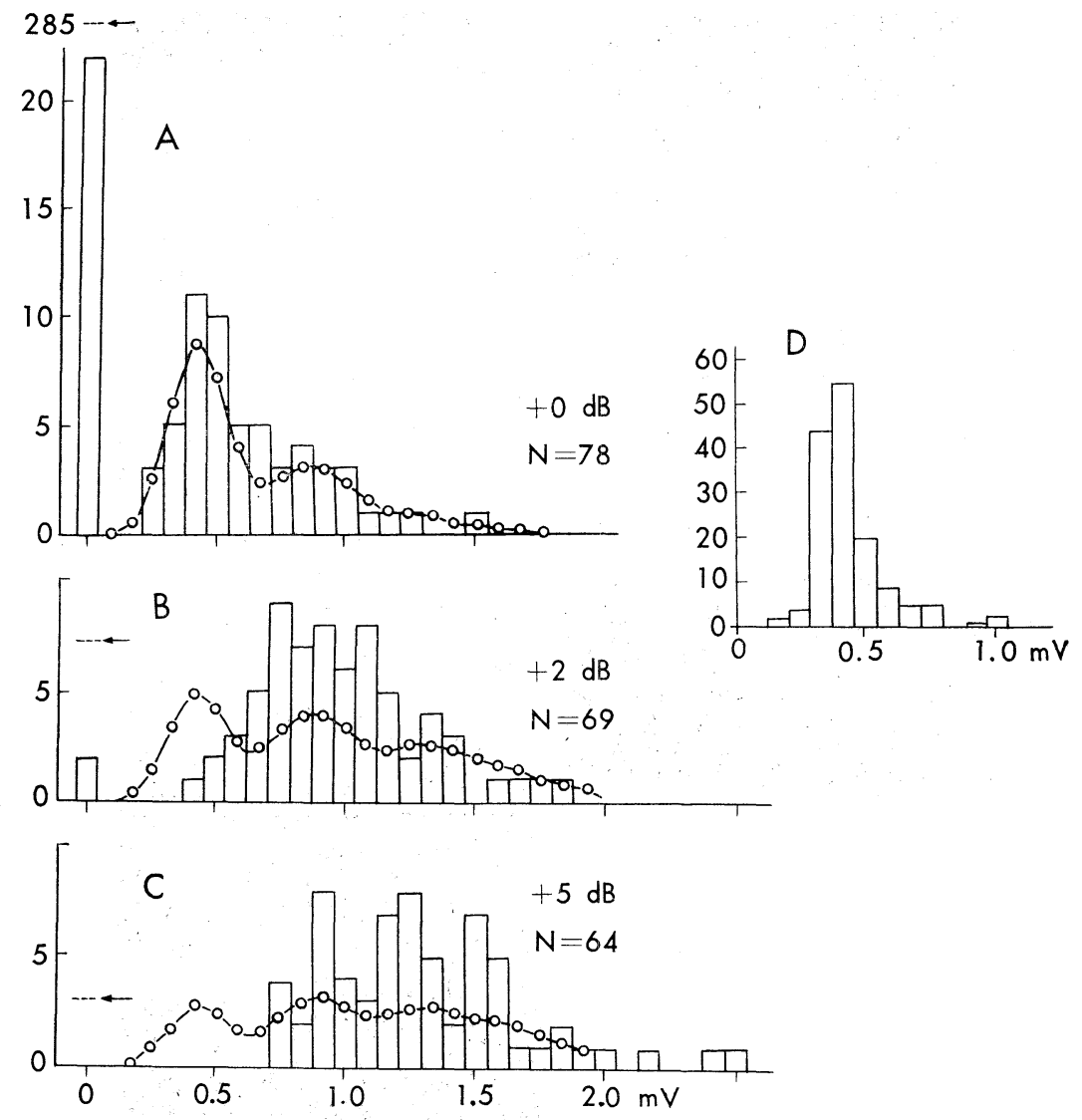

Fig. 4. A, B, and C: A comparison of expected distribution of EPSP size with those observed. Histograms show observed amplitude distributions of the first EPSP's for different intensities of sound. Sample records for A, B, and C are shown in Fig. 3 C1, $\mathrm{C} 2$ and $\mathrm{C} 3$, respectively. Smooth curves connecting open circles are expected amplitude distributions calculated for $m$ of 1.04, 2.25 and 3.07 for $\mathrm{A}, \mathrm{B}$, and C, respectively. $v_{1}$ and $\sigma$ were 0.43 and $0.11 \mathrm{mV}$, respectively. $\quad \mathrm{D}$ : Amplitude distribution of spontaneous miniature EPSP's shown in Fig. 3A.

to decide whether a given deflection might represent a true response or not. As a compromise, the response size was measured between two arrows shown in Fig. $3 \mathrm{Cl}$. The histogram shown in Fig. 4A illustrates results of 78 measurements. The mean quantum content $m$, being calculated from the equation (1), was found to be 1.04. Then the expected number of failures, single unit responses, double unit responses, and so forth were calculated from the Poisson theorem:

$$
n_{x}=\frac{N m^{x} e^{-m}}{x !}
$$


where $N=78, m=1.04$, and $x=0,1,2, \ldots$ Next, the expected distributions of $n_{1}, n_{2}$, and so forth were plotted. Namely, the distribution of $n_{1}$ was drawn around the mean of $v_{1}\left(\right.$ i.e., $0.43 \mathrm{mV}$ ) with a variance of $\sigma^{2}$, that of $n_{2}$ around the mean of $2 v_{1}$ with the variance of $2 \sigma^{2}$, and so forth. The sum of these individual curves would constitute the expected distribution. This is shown in Fig. 4A with the line connecting open circles. The expected number of failures, $n_{0}$, was 28.5 , a little greater than the actual value of 22 . Also the expected distribution curve is in fairly good agreement with the histogram experimentally obtained. Results of similar analysis applied to the experiments of Fig. 3 C2 and C3 are shown in Fig. 4B and C, respectively. In these two cases, the calculated distribution did not fit the observation. The mean quantum content was estimated too low in both of them. This seems to indicate that the Poisson theorem has only a limited applicability to the present case ( $c f$. Fig. 6), although the discrepancies may be partly attributable to a temporal dispersion and non-linear summation of unit EPSP's, etc. (KunO and MiYahaRA, 1969).

A different approach was employed for the analysis of the experiment shown in Fig. 2B. In this case the occurrence of spontaneous EPSP's was so infrequent that it was practically impossible to find the mean quantum content from the equation (1). Therefore, the mean quantum content was calculated with the following equation:

$$
m=\log _{e} \frac{\text { number of stimulus }(\mathrm{N})}{\text { number of failures of EPSP responses }\left(n_{0}\right)}
$$

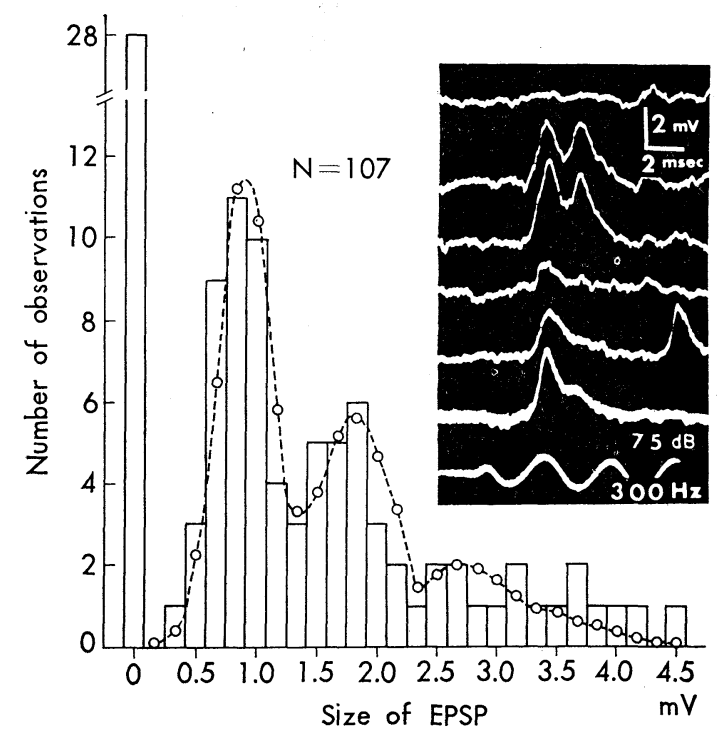

Fig. 5. Amplitude distribution of evoked EPSP's calculated using $m$ derived from the number of failures. $m: 1.34 \mathrm{mV} ; v_{1}: 0.89 \mathrm{mV} ; \sigma: 0.22 \mathrm{mV}$. See text for further details. 

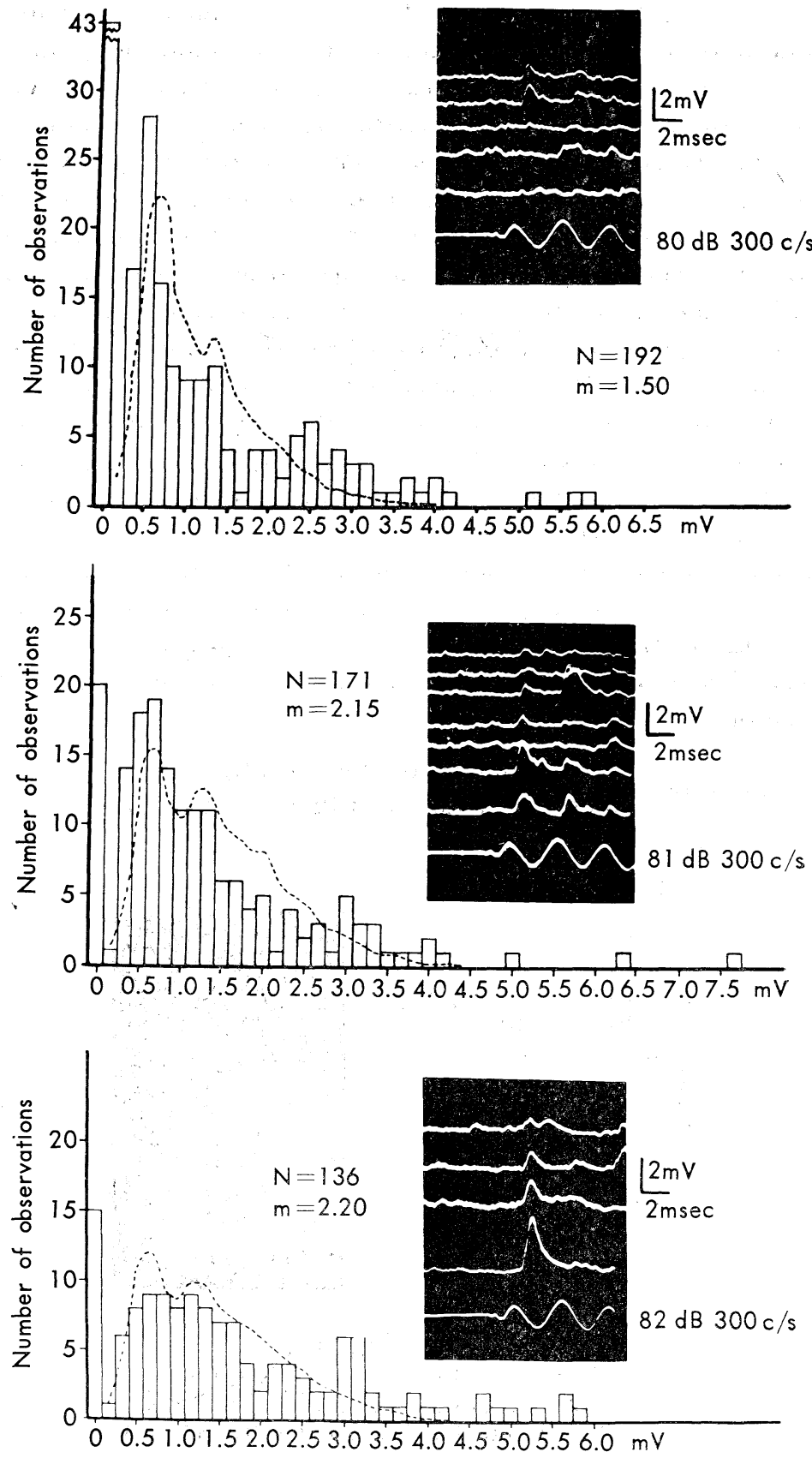

Fig. 6. Another instance in which amplitude distributions were calculated using $m$ 's derived from the number of failures. $v_{1}: 0.60 \mathrm{mV} ; \sigma: 0.20 \mathrm{mV}$. 
Using the values of $m$ thus obtained, the mean quantum size $v_{1}$ was obtained from the relation:

$$
v_{1}=\frac{\bar{v}}{m}
$$

As in the previous example, the expected figures for $n_{1}, n_{2}, n_{3}$, and so forth were calculated using equation (2). However, the standard deviation of the miniature EPSP's $\sigma$ is not known. Therefore, in drawing the Gaussian curve for $n_{1}$, the variance $\sigma^{2}$ was obtained by a trial and error method. The Gaussian curves for the second and the third groups were then drawn about the mean of $v_{2}$ and $v_{3}$, using $2 \sigma^{2}$, and $3 \sigma^{2}$ as their variances. The theoretical distribution curve which was obtained by adding four Gaussian curves together fits again fairly well with the histogram obtained experimentally shown in Fig. 5. In this case, the mean quantum content $m$, the quantum size $v_{1}$, and the standard deviation $\sigma$ were 1.34 , 0.89 , and $0.22 \mathrm{mV}$, respectively. The stimulus sound was $300 \mathrm{~Hz}$ at $75 \mathrm{~dB}$ SPL.

Figure 6 shows the same type of analysis employed in Fig. 5. The stimulus sound at $300 \mathrm{~Hz}$ was varied in its intensity from 80 to $82 \mathrm{~dB}$ by $1 \mathrm{~dB}$ steps. As expected, EPSP's increased in size with an increase in the sound intensity. Mean quantum content calculated separately using equation (3) turned out to be 1.50 , 2.15 , and 2.20, respectively. The theoretical distribution curves were drawn by employing common values of $v_{1}=0.60 \mathrm{mV}$ and $\sigma=0.20 \mathrm{mV}$. As can be seen in the figure, all the curves showed fairly good agreement with the histograms experimentally obtained.

\section{DISCUSSION}

Due to technical difficulties involved, the number of cases in which successful analysis could be performed was very limited in the present experiment. But the results described above seem to be consistent with the idea that the EPSP's in the eighth nerve fiber of goldfish are built up of all-or-none units which are identical in size and shape with the spontaneously occurring unit potentials. However, there are some problems to be considered.

The first one is concerned with the organization of the synaptic connection. Each eighth nerve fiber branches at its terminal region to innervate number of hair cells. But hair cells innervated by a single afferent fiber do not necessarily perform identical actions. We already know that hair cells of opposite spatial orientations are innervated by a single S1 fiber (Furukawa and ISHII, 1967). As for quantitative aspects, hair cells innervated by a single afferent fiber may be different in their sensitivity to sound and in their ability to evoke EPSP's. Also it is possible that EPSP's produced at individual nerve terminals suffer different amount of electrotonic decrement before reaching the recording site. From a practical point of view, however, these possible differences did not present any serious 
obstacle for the statistical treatments, as indicated, for example, by the fact that the amplitude of the spontaneously occurring miniature EPSP's distributed normally around the peak of the mean amplitude without disclosing multiple peaks.

The second problem concerns the mechanism of quantal release of the transmitter substance. It is generally admitted that a single presynaptic impulse releases only a fraction of the so-called immediately available store of transmitter substance from nerve terminals, as can be expressed in the following equation:

$$
m=n p
$$

where $m$ is the quantal content of the response, $n$ is the number of quanta in the readily available store, and $p$ is the fraction of the release (CHRISTENSEN and Martin, 1970; Martin, 1965). Usually, the applicability of the Poisson theorem is tested on preparations in which $m$ is made very small. The condition can be attained by reducing the $\mathrm{Ca}^{2+}$ concentration of the medium or by increasing its $\mathrm{Mg}^{2+}$ concentration or by a combination of both. It has been established that these procedures reduce $m$ by making $p$ small, while $n$ remains large (Del CaSTILLo and KATZ, 1954; KATZ and MiLedi, 1967). In the present experiment, however, we took advantage of the fact that the presynaptic elements are sensory hair cells, and applied very weak sound stimulus for obtaining small EPSP's. A difficulty here is that it has not been settled yet whether the magnitude of the presynaptic depolarization should affect $n$ or $p$. If it is as imed that $p$ is affected, there would arise no special problem at all, for the results could be interpreted basically in the same term as in other instances in which the modification of $m$ was attained by adjusting $\mathrm{Ca}^{2+}-\mathrm{Mg}^{2+}$ concentrations. But there are some indications which seem to suggest that the stimulus intensity, i.e., the mgnitude of the presynaptic depolarization, and $\mathrm{Ca}^{2+}-\mathrm{Mg}^{2+}$ concentrations in the medium are quite different in their mode of action, although the amount of the transmitter release may be modified by either of these factors. More concretely, our impression is that changes in the stimulus intensity interfere with the release by changing $n$, for the results on the "early tetanic run down" of EPSP's (ELMQVIST and QUASTEL, 1965) showed that $p$ remained at a constant value of about 0.6 (mean of 12 measurements; range being 0.73-0.52) for different stimulus intensities (FURUKAWA and MATSUURA, in preparation). A similar notion that the magnitude of the presynaptic depolarization may affect $m$ by changing $n$ has been expressed by HuBBARD and WiLLIS (1968).

Why is it then that the Poisson theorem could be applied, though to a limited extent, to our present case? We tentatively postulate the presence of multiple release sites in presynaptic cells each with different sensitivity to a depolarization. We assume that the transmitter is released at each site when the membrane depolarization exceeds the threshold for that site. As suggested by the fact that the curves relating the amount of release to the amplitude of the presynaptic depolarization are sigmoid in shape (IsHI et al., 1971), the threshold at the majority of 
release sites lies within a rather narrow range. Therefore, if it could be assumed that the thresholds at these large number of release sites show some random temporal variations around their equilibrium positions, our experiments meet the Poisson condition with only a very small number responding each time out of a large population of release sites.

We would like to express our thanks to Dr. A. Kitabatake for his helpful suggestions regarding statistical studies.

\section{REFERENCES}

Christensen, B. N. and Martin, A. R. (1970) Estimation of probability of transmitter release at the mammalian neuromuscular junction. J. Physiol., 210: 933-945.

Del Castillo, J. and Katz, B. (1954) Quantal components of the end-plate potential. $J$. Physiol., 124: 560-573.

ElmQvist, D. and Quastel, D. M. J. (1965) A quantitative study of end-plate potentials in isolated human muscle. J. Physiol., 178: 505-529.

Furukawa, T. and IsHII, Y. (1967) Neurophysiological studies on hearing in goldfish. $J$. Neurophysiol., 30: 1377-1403.

Furukawa, T., IshiI, Y. and Matsuura, S. Synaptic delay and time course of EPSP's at the junction between hair cells and eighth nerve fibers in the goldfish. (in preparation)

FurukAwA, T. and Matsuura, S. Adaptation of sound-evoked EPSP's in auditory fibers of goldfish. (in preparation)

HubBard, J. I. and WiLLIS, W. D. (1968) The effects of depolarization of motor never terminals upon the release of transmitter by nerve impulses. J. Physiol., 194: 386-405.

IshiI, Y., MatsuURA, S. and FuruKawa, T. (1971) An input-output relation at the synapse between hair cells and eighth nerve fibers in goldfish. Jap. J. Physiol., 21: 91-98.

KatZ, B. (1966) In Nerve, Muscle, and Synapse. McGraw-Hill, New York.

KATZ, B. and MILEDI, R. (1967) The timing of calcium action during neuromuscular transmission. J. Physiol., 189: 535-544.

Kuno, M. and Miyahara, J. T. (1969) Non-linear summation of unit synaptic potentials in spinal motoneurones of the cat. J. Physiol., 201: 465-477.

Martin, A. R. (1965) Quantal nature of synaptic transmission. Physiol. Rev., 46: 51-66. 